\title{
PERSPECTIVES IN HIGH-ENERGY PHYSICS
}

\author{
John Ellis \\ Theoretical Physics Division, CERN, CH-1211 Geneva 23 \\ E-mail: John.Ellis@cern.ch
}

Abstract: A personal view of current prospectives in particle physics is presented, inspired by the contributions to this meeting. Particular emphasis is laid in precision tests of the Standard Model and the search for the Higgs boson, on probes of CP violation, on speculations about possible physics beyond the Standard Model, on neutrino masses and oscillations, on the quest for supersymmetry, on opportunities @ future accelerators, and on the ultimate phenomenological challenge offered by the quest for a Theory of Everything.

CERN-TH/2000-129

\section{The Standard Model}

The Standard Model continued to rule accelerator experiments during 1999, even as the heroic efforts of the CERN accelerator engineeers pushed the LEP centre-of-mass energy to $202 \mathrm{GeV}$, and briefly to $204 \mathrm{GeV}$. There were no surprises in fermion-pair production or in the bread-and-butter reaction of LEP2, $e^{+} e^{-} \rightarrow W^{+} W^{-}$. Both the $\gamma W^{+} W^{-}$and $Z^{0} W^{+} W^{-}$triple-gauge-boson vertices are there, as seen in Fig. 1, with magnitudes close to the Standard Model values [1, 2]. Looking into the final states, there are no confirmed interferences in $\left(W^{+} \rightarrow \bar{q} q\right) \otimes\left(W^{-} \rightarrow \bar{q} q\right)$ final states, due to either colour rearrangement or Bose-Einstein correlations: the difference between the $W$ mass measured in purely hadronic and other final states is $15 \pm 55 \mathrm{MeV}$ [3]. Combining all the LEP measurements, one finds [1]

$$
m_{W}=80.401 \pm 0.048 \mathrm{GeV}(\mathrm{LEP})
$$

contributing with the hadron colliders $\left(M_{W}=\right.$ $80.448 \pm 0.062 \mathrm{GeV})$ to a global average

$$
m_{W}=80.419 \pm 0.038 \mathrm{GeV} \text { (world). }
$$

This error is now comparable with the value estimated indirectly from precision electroweak mea- hep-ph/0007161

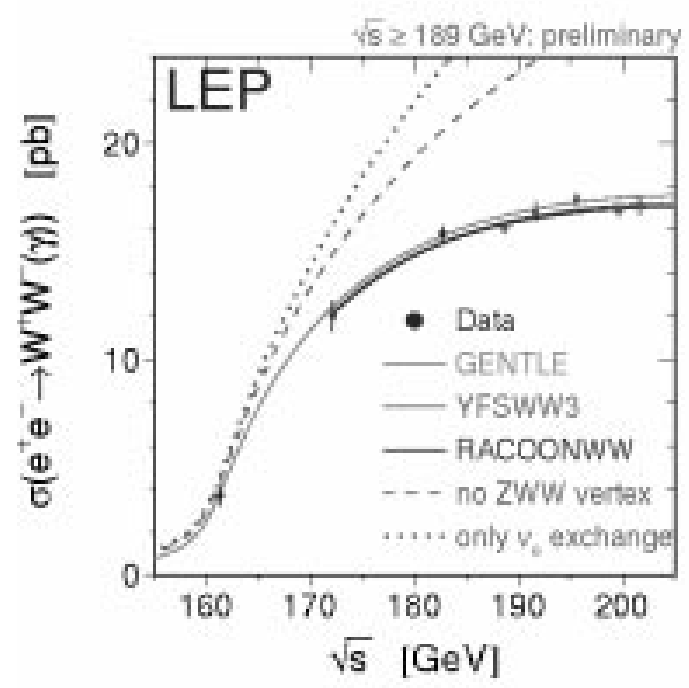

Figure 1: The Standard Model rules OK: measurements of $\sigma\left(e^{+} e^{-} \rightarrow W^{+} W^{-}\right)$at LEP 2 agree with theory, demonstrating the existence of the expected $\gamma W^{+} W^{-}$and $Z^{0} W^{+} W^{-}$vertices [1].

surements: $m_{W}=80.382 \pm 0.026 \mathrm{GeV}$, provides new, independent evidence for a light Higgs boson, and begins to impact significantly the radiativecorrection estimate [1]

$$
m_{H}=77_{-39}^{+69} \mathrm{GeV}
$$


when $\alpha_{e m}\left(m_{Z}\right)^{-1}=128.878 \pm 0.090$ is assumed (or $\log \left(M_{H} / \mathrm{GeV}\right)=1.96_{-0.23}^{+0.21}$ if the estimate $128.905 \pm 0.036$, with the error reduced by theory, is assumed). The Higgs boson probably weighs less than $200 \mathrm{GeV}$.

The plan is to raise the LEP energy as high as possible during 2000, with the primary aim of searching for the Higgs boson. An integrated luminosity of $50 / \mathrm{pb}$ per experiment at $206 \mathrm{GeV}$ would increase the sensitivity of the Higgs reach from the current lower limit of $107.9 \mathrm{GeV}[4,5]$ to about $114 \mathrm{GeV}$ [6]. A small numerical increase, but in the most interesting range, also from the point of view of supersymmetry [7]. The LEP energies attained so far range up to $208.7 \mathrm{GeV}$, with a total luminosity (so far) of $109 \mathrm{pb}^{-1}$ at an average energy above $205 \mathrm{GeV}$. It seems that the target sensitivity to $m_{H}=114 \mathrm{GeV}$ is well within reach. At the time of writing, the current sensitivity is to $m_{H} \sim 113.4 \mathrm{GeV}$, and the latest update may be obtained from [8].

Then, in Autumn 1999, LEP must be shut down and dismantled to make way for the LHC excavations and installation. It will be the end of an era of precision electroweak measurements. The search for the Higgs boson will then pass to Fermilab, where the Tevatron has a chance of exploring higher Higgs masses if it gathers more than $10 \mathrm{fb}^{-1}$ of luminosity, as seen in Fig. 2 [10].

\section{CP Violation}

For 24 years, it was possible to think that the CP violation [11] seen in the $K^{0}-\bar{K}^{0}$ system might be due entirely to a superweak force beyond the Standard Model, inducing CP violation in the $K^{0}-\bar{K}^{0}$ mass matrix: $\operatorname{Im} M_{12} \neq$ 0 . Thanks to the new measurements of $\epsilon^{\prime} / \epsilon$ in $1999[12,13]$, signalling direct $\mathrm{CP}$ violation in the $K^{0} \rightarrow 2 \pi$ decay amplitudes, we now know the superweak theory cannot be the whole story. The Standard Model with 6 quarks permits CP violation in the $W^{ \pm}$couplings [14], but there are no other sources if there is just one Higgs doublet. The MSSM contains many possible sources of $\mathrm{CP}$ violation, of which the most important for

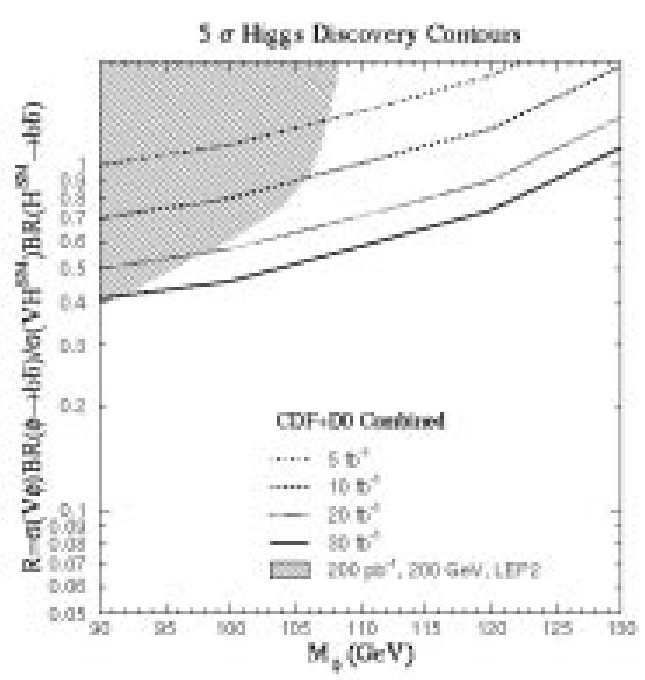

Figure 2: Higgs discovery sensivity of the Fermilab Tevatron collider compared with that of LEP [10].

hadron decays may be the phases of the trilinear soft supersymmetry-breaking couplings $A_{t, b}$ of the third generation and of the gluino mass, relative to the Higgs mixing parameter $\mu$.

In the Standard Model, one may estimate [15]

$$
\begin{gathered}
\operatorname{Re}\left(\frac{\epsilon^{\prime}}{\epsilon}\right) \simeq 13 \operatorname{Im} \lambda_{t}\left(\frac{130 \mathrm{MeV}}{m_{s}\left(m_{c}\right)}\right)^{2}\left(\frac{\Lambda^{(4)}}{340 \mathrm{MeV}}\right) \\
\times\left[B_{6}(1-\Omega)-0.4 B_{8}\left(\frac{m_{t}\left(m_{t}\right)}{165 \mathrm{GeV}}\right)^{2.5}\right]
\end{gathered}
$$

where $\operatorname{Im} \lambda_{t}$ is a Cabibbo-Kobayashi-Maskawa angle factor and $\Omega$ is an electroweak penguin effect. Calculating $\operatorname{Re}\left(\epsilon^{\prime} / \epsilon\right)$ accurately is difficult because of potential cancellations in (2.1), and the fact that the strong-interaction matrix elements $B_{6}$ and $B_{8}$ are relatively poorly known. A conservative estimate in the Standard Model would be that [15]

$$
1 \times 10^{-4}<\operatorname{Re}\left(\epsilon^{\prime} / \epsilon\right)<3 \times 10^{-3}
$$

The world data, including the new NA48 measurement reported here [16], average to

$$
\operatorname{Re}\left(\epsilon^{\prime} / \epsilon\right)=(19.3 \pm 2.4) \times 10^{-4}
$$

as seen in Fig. 3. This is compatible with the Standard Model range (2.2), although probably requiring a relatively big penguin matrix element $B_{6}$ : an emperor maybe? The measurement of 


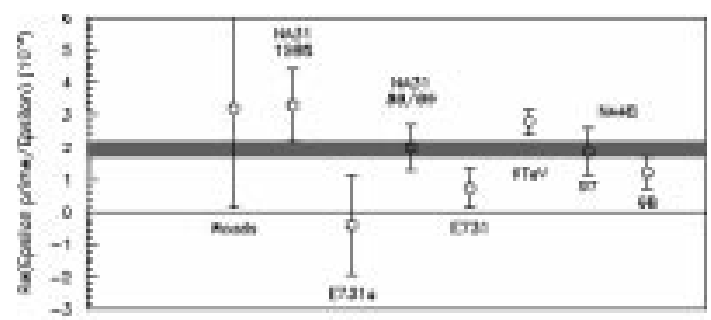

Figure 3: Compilation of measurements of $\operatorname{Re}\left(\epsilon^{\prime} / \epsilon\right)$ [16].

$\left(\epsilon^{\prime} / \epsilon\right)$ will be refined by further $\mathrm{KTeV}$ and NA48 data, and by KLEO at DAFNE.

If the relatively large value of $\left(\epsilon^{\prime} / \epsilon\right)$ has a large supersymmetric contribution, this could show up in $K_{L}^{0} \rightarrow \pi^{0} \bar{\nu} \nu, \pi^{0} e^{+} e^{-}$and $\pi^{0} \mu^{+} \mu^{-}$decays [17]. The present upper limits on these decays from $\mathrm{KTeV}$ are far above the Standard Model predictions, so here is a physics opportunity worth pursuing in parallel with $B$ experiments.

The main target of these experiments will be the Standard Model unitarity triangle shown in Fig. 4 [18], with the hope of finding a discrepancy: perhaps it will turn out to be a quadrilateral? The poster child for the dawning $\mathrm{CP}$ age is the measurement of $\sin 2 \beta$ via $B^{0} \rightarrow J / \psi K_{S}$ decay. The theory is gold-plated - with penguin pollution expected only at the $10^{-3}$ level - and the experiment is clean. Indeed, between them, OPAL [19], CDF [20] and ALEPH [21] have almost measured it:

$$
\sin 2 \beta=0.91 \pm 0.35
$$

A new era of precision flavour physics is now dawning, with the $B$ factories now starting to take data. They should reduce the error in $\sin 2 \beta$ below 0.1 , and the LHC experiments aim at an error $\sim 0.01$.

That is the good news: the bad news concerns the measurement of $\sin 2 \alpha$ via $B^{0} \rightarrow \pi^{+} \pi^{-}$ decay. This mode has now been seen by CLEO [22], but with a relatively low branching ratio

$$
B\left(\pi^{+} \pi^{-}\right)=\left(0.43_{-0.14}^{+0.16} \pm 0.05\right) \times 10^{-5}
$$

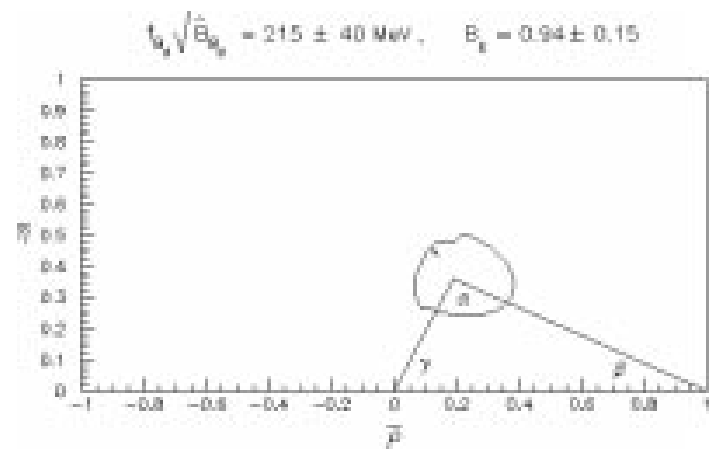

Figure 4: The result of a recent global fit to the Cabibbo-Kobayashi-Maskawa parameters [18].

whereas

$$
B\left(K^{+} \pi^{-}\right)=\left(1.72_{-0.24}^{+0.25} \pm 0.12\right) \times 10^{-5}
$$

This is bad news because it suggests that penguin pollution is severe, and difficult to disentangle, as well as threatening a large background. This worry has revived interest in the study of $\sin 2 \alpha$ via $B^{0} \rightarrow \rho^{ \pm} \pi^{\mp}$ and other decays. There is no time here to review proposed strategies for measured $\gamma$ : suffice to say that within a decade we may know the sum $\alpha+\beta+\gamma$ with a precision $\sim 10^{0}$. Let us hope that it turns out to be inconsistent with $180^{\circ}$ !

\section{Beyond the Standard Model}

The Standard Model has many defects, the most severe being that it agrees with all accelerator data. Nevertheless, it is very unsatisfactory, in that it provides no explanations for the particle quantum numbers $\left(Q_{e m}, I, Y\right.$, colour), and contains 19 arbitrary parameters: 3 independent gauge couplings, $1 \mathrm{CP}$-violating strong-interaction phase, 6 quark masses, 3 charged-lepton masses, 4 Cabibbo-Kobayashi-Maskawa mixing parameters, the $W$ mass and the Higgs mass.

As if this was not enough, the indications of neutrino oscillations introduce at lest 9 more parameters: 3 neutrino masses, 3 neutrino mixing angles and $3 \mathrm{CP}$-violating phases, without even talking about the mechanism for neutrino mass generation. Also, we should not forget gravity, with Newton's constant and the cosmologi- 
cal constant as two new parameters, at least one more parameter to generate the baryon asymmetry of the Universe, at least one more to describe cosmological inflation, etc.

It is common to group the open problems beyond the Standard Model into three categories: Mass - do the particle masses indeed originate from a Higgs boson, and is this accompanied by supersymmetric particles? Flavour - why are there so many flavours of quarks and leptons, and what explains the ratios of their masses and weak mixings? and Unification - is there a simple group structure containing the strong, weak and electromagnetic interactions? Beyond these beyonds lurks the quest for a Theory of Everything that should also include gravity, reconcile it with quantum mechanics, explain the origin of space-time and why it has 4 dimensions, and make Colombian coffee. This is the ambition of string theory and its latest incarnation as $M$ theory.

Subsequent sections of this talk deal with these ideas and how they may be tested.

\section{Neutrino Masses and Oscillations}

Why not? Although the Standard Model predicts $m_{\nu}=0$ if one ignores possible non-renormalizable interactions, there is no deep reason why this should be so. Theoretically, we expect masses to vanish only if there is a good asymmetry reason, in the form of an exact gauge symmetry. For example, the $U(1)$ gauge symmetry of QED guarantees the conservation of electric charge and the masslessness of the photon. However, there is no exact gauge symmetry or massless gauge boson coupled to lepton number $L$, which we therefore expect to be violated. Neutrino masses are possible if there is an effective $\Delta L=2$ interaction of the Majorana form $m_{\nu} \nu \cdot \nu$. Such interactions are generic in Grand Unified Theories (GUTs) with their extra particles, but could even be fabricated from Standard Model particles alone [23], if one allows non-renormalizable interactions of the form $\frac{1}{M} \nu H \cdot \nu H \rightarrow m_{\nu}=<0|H| 0>^{2} / M$, where $M$ is some heavy mass scale: $M \gg m_{W}$.
Nevertheless, generic neutrino mass terms, as they arise in typical GUTs, have the seesaw form $[24]$

$$
\left(\nu_{L}, \nu_{R}\right)\left(\begin{array}{cc}
0 & m \\
m^{T} & M
\end{array}\right)\left(\begin{array}{c}
\nu_{L} \\
\nu_{R}
\end{array}\right)
$$

where the $\nu_{R}$ are singlet "right-handed" neutrinos. After diagonalization, (4.1) yields

$$
m_{\nu}=m \frac{1}{M} m^{T}
$$

which is naturally small: $m_{\nu} \ll m_{q, \ell}$, if $m \sim m_{q, \ell}$ and $M=\mathcal{O}\left(M_{G U T}\right)$. For example, if $m \sim 10$ $\mathrm{GeV}$ and $M \sim 10^{13} \mathrm{GeV}$, one finds $m_{\nu} \sim 10^{-2}$ $\mathrm{eV}$, in the range indicated by experiments on solar and atmospheric neutrinos. Each of the fields $\left(\nu_{L}, \nu_{R}\right)$ in (4.1) should be regarded as a threedimensional vector in generation space, so $m_{\nu}$ (4.2) is a $3 \times 3$ matrix. its diagonalization $V_{\nu}$ relative to that of the charged leptons $V_{\ell}$ yields the Maki-Nakagawa-Sakata neutrino mixing matrix $V_{M N S}=V_{\ell} V_{\nu}^{+}$between the interaction eigenstates $\nu_{e, \mu, \tau}[25]$.

Could there be additional light neutrinos? The LEP neutrino counting measurements tell us that these could only be sterile neutrinos $\nu_{s}$. But what would prevent them from acquiring large masses $m_{s} \nu_{s} \nu_{s}: m_{s} \gg m_{W}$, since they have no electroweak quantum numbers to forbid them via selection rules? This is exactly what happens to the $\nu_{R}$ in (4.1). Most theorists expect the observed neutrinos $\nu_{\text {light }} \sim \nu_{L}$, and their effective mass term to be of the Majorana type. Before the advent of the atmospheric neutrino data, most theorists might have favoured small neutrino mixing angles, by analogy with the CKM mixing of quarks. But this is not necessarily the case, and many plausible models have now been constructed in which the neutrino mixing is large, because of large mixing in the light-lepton sector $V_{\ell}$ and/or in the heavy Majorana mass matrix $M$ and hence $V_{\nu}$.

In hierarchical models of neutrino models, as often arise in GUTs: $m_{1}^{2} \gg m_{2}^{2} \gg m_{3}^{2}$, one may interpret $\Delta m^{2}$ as $m_{\nu_{H}}^{2}$, the squared mass of the heavier eigenstate in the oscillation. Cosmological data exclude $m_{\nu} \gtrsim 3 \mathrm{eV}$, but even $m_{\nu} \sim$ $0.03 \mathrm{eV}$ may be of cosmological importance [26]. 
Thus, although the unconfirmed LSND signal would certainly be of cosmological interest, so also are the confirmed atmospheric neutrino data.

Even the solar neutrino data become of interest to cosmology if the three neutrino flavous are almost degenerate, with $\Delta m^{2} \ll m$, a possibility that cannot be excluded by the oscillation data. Such a degeneracy is constrained by the upper limit on neutrinoless $\beta \beta$ decay [27]:

$$
<m_{\nu}>_{e} \lesssim 0.2 \mathrm{eV}
$$

where the expectation value is weighted by the neutrinos' electron couplings. The limit (4.3) is compatible with the neutrino masses close to the cosmological upper limit (which nearly coincides with the direct upper limit on $m_{\nu_{e}}$ from the end-point of Tritium $\beta$ decay) if there is almost maximal neutrino mixing. There is an issue whether this can be maintained in the presence of the mass renormalization expected in GUTs, which tend to break the mass degeneracy and cause mixing to become non-maximal [28]. However, these difficulties may be avoided in some models of neutrino flavour symmetries [29].

As was discussed here by Smirnov [30] and is seen in Fig. 5, the best fit to the Super-Kamiokande atmospheric-neutrino data is with near-maximal mixing and $\Delta m^{2} \simeq 3.7 \times 10^{-3} \mathrm{eV}$, the $90 \%$ confidence-level range being $(2$ to 7$) \times 10^{-3} \mathrm{eV}^{2}[31]$. This is compatible with the $90 \%$ confidencelevel ranges favoured by the other atmosphericneutrino experiments (Kamiokande, MACRO and Soudan). As for the solar-neutrino data, there have been four favoured regions, three of which shown in Fig. 6: the large- and small-mixingangle MSW solutions (LMA and SMA) with $\Delta m^{2} \sim$ $10^{-5} \mathrm{eV}^{2}$, another MSW solution with lower $\Delta m^{2} \sim$ $10^{-7} \mathrm{eV}^{2}$ (LOW), and vacuum solutions (VAC) with $\Delta m^{2} \sim 10^{-9}$ to $10^{-11} \mathrm{eV}^{2}[32]$.

How may one discriminate between the different oscillation scenarios? We know already from Super-Kamiokande that $\nu_{\mu} \rightarrow \nu_{e}$ atmospheric oscillations are not dominant, and more stringent upper limits are imposed by the Chooz and Palo Verde reactor experiments [33]. However, $\nu_{\mu} \rightarrow$ $\nu_{e}$ oscillations may be present at a lower level: if so, they may enable the sign of $\Delta m^{2}$ to be deter-

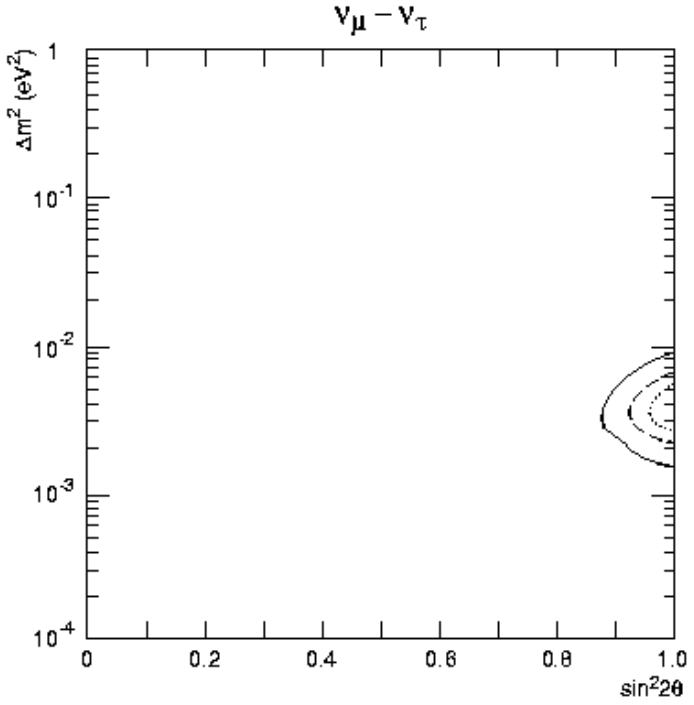

Figure 5: Regions of neutrino oscillation parameter space favoured by data on atmospheric neutrinos [31].

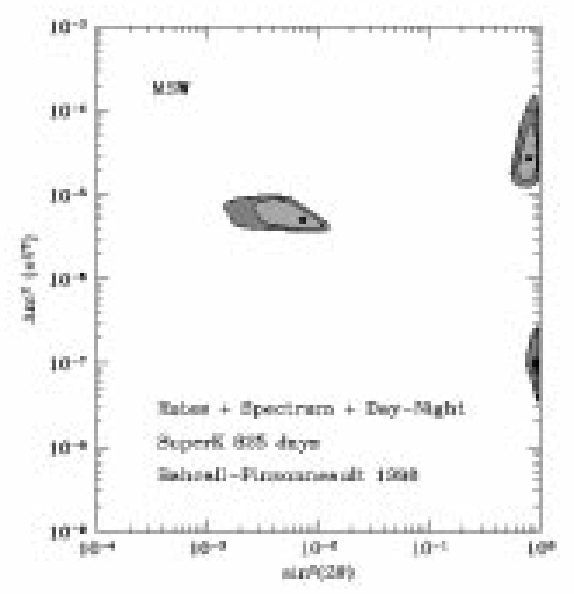

Figure 6: $M S W$ fits to the solar neutrino data [32].

mined via matter effects. There are several ways to distinguish between dominance by $\nu_{\mu} \rightarrow \nu_{\tau}$ or $\nu_{\mu} \rightarrow \nu_{s}$. The zenith-angle distributions in various categories of Super-Kamiolande events (highenergy partially-contained events, throughgoing muons and neutral-current enriched events) favour $\nu_{\mu} \rightarrow \nu_{\tau}$ oscillations, now at the $99 \%$ confidence level [34]. Another way to distinguish $\nu_{\mu} \rightarrow$ $\nu_{\tau}$ from $\nu_{\mu} \rightarrow \nu_{s}$ is $\pi^{0}$ production in SuperKamiokande, which is impossible in $\nu_{s}$ interactions. The present data appear to favour $\nu_{\mu} \rightarrow$ 
$\nu_{\tau}$, but, before reaching a conclusion, it will be necessary to reduce the systematic error in the production rate, which should be possible using data from the $\mathrm{K} 2 \mathrm{~K}$ near detector.

As for solar neutrinos, no significant distortion in the energy spectrum is now observed by Super-Kamiokande [35], and there is an upper limit on the flux of hep neutrinos that excludes the possibility that they might influence oscillation interpretations. Moreover, the day-night effect is now apparent only at the 1.3- $\sigma$ level, and the seasonal variation that seems to be emerging is completely consistent with the expected geometric effect of the Earth's orbital eccentricity. These observations are all consistent with the LMA interpretation if $\Delta m^{2} \gtrsim 2 \times 10^{-3} \mathrm{eV}^{2}$, but the constraints from the day and night spectra now disfavour the SMA and VAC interpretations at the $95 \%$ confidence level [35]. Moreover, $\nu_{e} \rightarrow \nu_{s}$ is also disfavoured at the same level. Super-Kamiokande seems to be pushing us towards the LMA $\nu_{\mu} \rightarrow \nu_{\tau}$ interpretation, but has not yet provided a 'smoking gun' for this interpretation.

In the near future, SNO will be providing information on the neutral-current/charged-current ratio, discriminating between the different solar-neutrino scenarios and telling us whether the $\nu_{e}$ have oscillated into $\nu_{\mu} / \nu_{\tau}$ or $\nu_{s}$ [36]. In the longer term, BOREXINO will check the disappearance of the ${ }^{7} \mathrm{Be}$ solar neutrinos [37].

Many of the most important prospective developments may be provided by long-baseline terrestrial neutrino experiments. The first of these is $\mathrm{K} 2 \mathrm{~K}$, which has already released some preliminary results [38]. They see 17 fully-contained events in their fiducial region, whereas $29.2_{-3.3}^{+3.5}$ would have been expected in the absence of oscillations, versus $19.3_{-2.4}^{+2.5}$ if $\delta m^{2}=3 \times 10^{-3}$. Overall, they see a total of 44 events, whereas about 74 (50) would have been expected in all event categories in the absence of oscillations (if $\delta m^{2}=$ $3 \times 10^{-3}$ ), A detailed fit including data from the run currently underway and energy-spectrum information is now being prepared, but the present data already appear to be very promising! In a few years' time, KamLAND will use reactor neu-

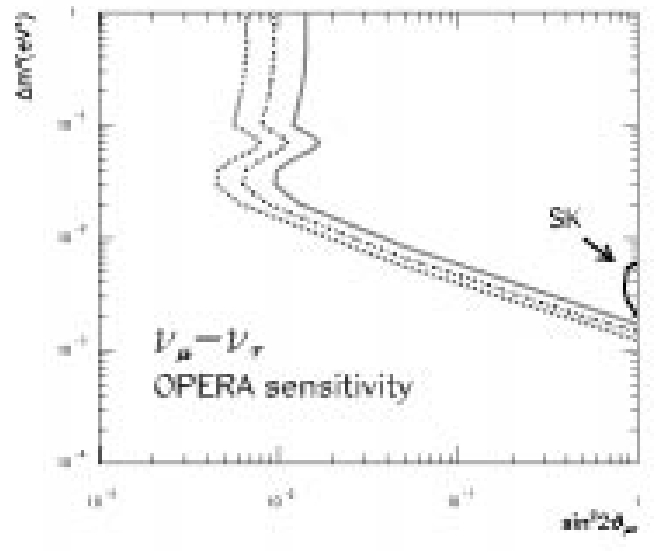

Figure 7: Sensitivity to $\nu_{\mu} \rightarrow \nu_{\tau}$ oscillations of the OPERA experiment [42] proposed for the CERNGran Sasso neutrino beam [41].

trinos to check the LMA MSW solution to the solar-neutrino deficit [39]. Starting probably in 2003, MINOS will be looking for $\nu_{\mu}$ disappearance, measuring the neutral-current/charged-current ratio, and looking for $\nu_{e}$ appearance [40].

Then, starting in 2005, the CERN-Gran Sasso project will provide the opportunity to look for $\nu_{\mu} \rightarrow \nu_{\tau}$ oscillations directly via $\tau$ production [41]. This seems to me a key experiment: "If you have not seen the body, you have not proven the crime." The beam energy has been optimized for $\tau$ production, and either of the proposed experiments (OPERA [42] and ICANOE [43]) should be able to discover $\tau$ production at the 4- $\sigma$ level if the atmospheric-neutrino parameters are in the range favoured by Super-Kamiokande, as exemplified in Fig. 7. Additionally, ICANOE [43] may be able to probe the LMA MSW solar solution via low-energy atmospheric-neutrino events.

\section{Supersymmetry}

As you know, the motivation for supersymmetry at accessible energies is the hierarchy problem [44], namely why $m_{W} \ll m_{P}$, or equivalently why $G_{F} \gg G_{N}$, or equivalently why the Coulomb potential dominates over the Newton potential in atoms. Even if the hierarchy is set 
by hand, an issue is raised by the quantum corrections:

$$
\delta m_{H, W}^{2}=\mathcal{O}\left(\frac{\alpha}{\pi}\right) \Lambda^{2} \gg m_{W}^{2}
$$

where $\Lambda$ is a cutoff reflecting the appearance of new physics beyond the Standard Model. Supersymmetry is a favoured example, making the corrections (5.1) naturally small:

$$
\delta m_{H, W}^{2}=\mathcal{O}\left(\frac{\alpha}{\pi}\right) \quad\left|m_{B}^{2}-m_{F}^{2}\right|
$$

which is $\lesssim m_{H, W}^{2}$ if

$$
\left|m_{B}^{2}-m_{F}^{2}\right| \lesssim 1 \mathrm{TeV}^{2}
$$

for the difference in mass-squared between spartners. Circumstantial evidence for supersymmetry is provided by the concordance between the gauge coupling strengths measured at LEP and elsewhere with supersymmetric GUTs [45], and the indirect LEP indications for a light Higgs boson [1], as predicted by supersymmetry [7] and discussed shortly. However, neither these nor the naturalness/fine-tuning argument set rigorous upper bounds on the sparticle mass scale [46].

Another argument favouring low sparticle masses is provided by cold dark matter [47]. The lightest sparticle is commonly expected to be the lightest eigenstate of the neutralino mass matrix:

$$
\left(\begin{array}{cccc}
M_{2} & 0 & \frac{-g_{2} v_{2}}{\sqrt{2}} & \frac{g_{2} v_{1}}{\sqrt{2}} \\
0 & M_{1} & \frac{-g^{\prime} v_{2}}{\sqrt{2}} & \frac{g^{\prime} v_{1}}{\sqrt{2}} \\
\frac{-g_{2} v_{2}}{\sqrt{2}} & \frac{-g^{\prime} v_{1}}{\sqrt{2}} & 0 & \mu \\
\frac{-g_{2} v_{1}}{\sqrt{2}} & \frac{-g^{\prime} v_{1}}{\sqrt{2}} & \mu & 0
\end{array}\right)
$$

The gaugino masses $M_{2}, M_{1}$ are commonly assumed to be equal at the GUT scale:

$$
M_{2}=M_{1} \equiv m_{1 / 2}
$$

and are then renormalized: $M_{2} / M_{1} \simeq \alpha_{2} / \alpha_{1}$ at lower scales. The scalar masses may also be universal at the GUT scale, in which case they are also renormalized: $m_{0_{i}}^{2}=m_{0}^{2}+C_{i} m_{1 / 2}$, and ratio of Higgs v.e.v.'s is commonly denoted by $\tan \beta \equiv v_{2} / v_{1}$
The LEP limits on neutralinos and charginos [48] exclude the possibility that the lightest neutralino $\chi$ is an almost pure photino or Higgsino. If univerality is assumed, or if neutralinos constitute the bulk of the cold dark matter [49], a dominant $U(1)$ gaugino component is favoured, as shown in Fig. 8 [50]. As discussed in [50], one must take into account the various constraints on the universal parameters $\left(m_{1 / 2}, m_{0}\right)$ imposed by LEP, the absence of charged $\tilde{\tau}$ dark matter, the observed rate for $b \rightarrow s \gamma$ decay and the absence of a charge- and colour-breaking (CCB) vacuum. The allowed dark-matter region is stretched to large $m_{1 / 2}$ by neutralino-slepton coannihilation, which allow $m_{\chi} \lesssim 600 \mathrm{GeV}[51]$.

The most direct experimental constraints on the supersymmetric parameter space are those for charginos, neutralinos and sleptons at LEP, which impose $m_{\chi^{ \pm}} \gtrsim 100 \mathrm{GeV}$ and $m_{\tilde{e}} \gtrsim 90 \mathrm{GeV}$ generically. The Tevatron constraints on squarks and gluinos are of less direct importance if universality is assumed. However, stop searches at LEP and the Tevatron are important for constraining the radiative corrections to the lightest supersymmetric Higgs mass, whose leading terms contribute [7]

$$
\delta m_{h}^{2}=\mathcal{O}(\alpha) \frac{m_{t}^{4}}{m_{W}^{2}} \ln \left(\frac{m_{\tilde{t}}^{2}}{m_{t}^{2}}\right)
$$

These are relevant to the constraints on $m_{0}$ and $m_{1 / 2}$ imposed by the absence of a Higgs boson at LEP [51].

Fig. 9 displays the lower limits on $m_{\chi}$ imposed by all these constraints, either if scalarmass universality is (UHM) or is not (nUHM) assumed [51]. In the $\mathrm{UHM}_{\text {min }}$ scenario in Fig. 9, the absence of CCB vacua is not required. Fig. 9 also displays the expected impact of LEP searches in 2000, assuming pessimistically that they are unsuccessful. We see that

$$
m_{\chi} \gtrsim 50 \mathrm{GeV} \quad \text { and } \quad \tan \beta \gtrsim 3,
$$

with the precise values depending on the scenario adopted.

The above analysis assumed that $\mathrm{CP}$ violation could be ignored. However, as emphasized here by Kane [52], CP violation in the soft 


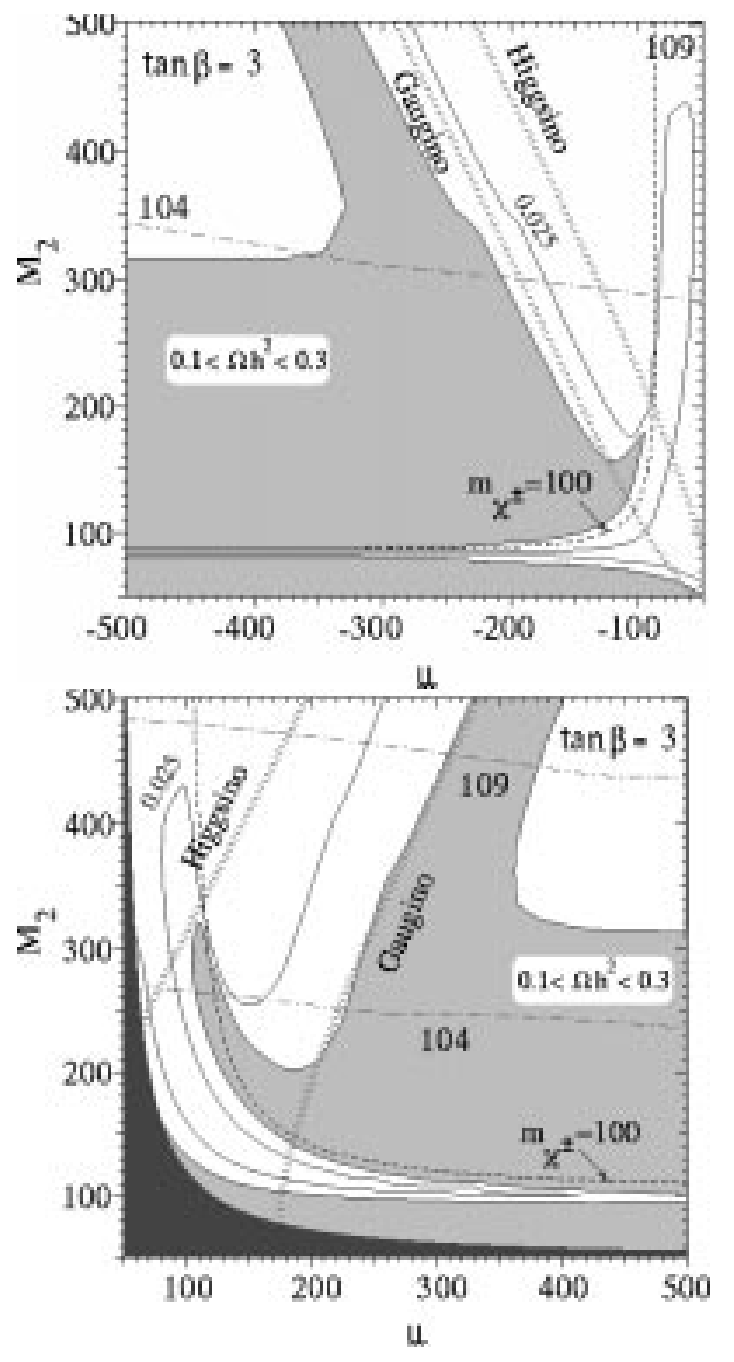

Figure 8: Regions of the $\mu, M_{2}$ plane where the lightest neutralino $\chi$ may constitute the cold dark matter [51] (light shading). In most of the allowed regions, the $\chi$ is mainly a $U(1)$ gaugino. The dark shaded region was excluded by LEP 1.

supersymmetry-breaking parameters may be important. Indeed, such CP violation is essential in electroweak baryogenesis [53 ${ }^{1}$, which also requires a first-order phase transition, and hence a relatively light Higgs boson and stop squark, as seen in Fig. 10 [55]! LEP had been thought almost to exclude such a scenario because of its lower limit on $m_{h}$. However, we have recently emphasized [56] that the LEP lower limit on $m_{h}$ may be greatly relaxed in the presence of $\mathrm{CP}$ vi-

\footnotetext{
${ }^{1}$ The popular alternative of leptogenesis was discussed here by Ma [54].
}
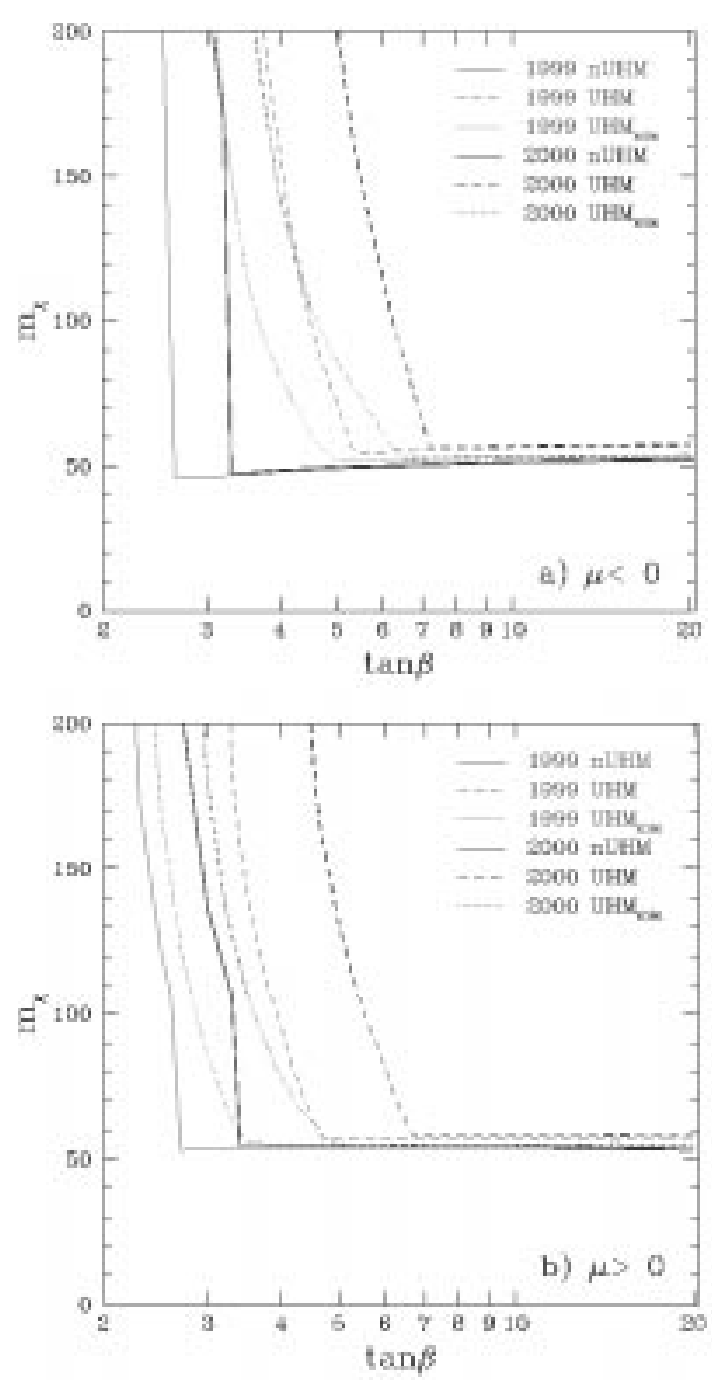

Figure 9: Lower limits on the mass of the lightest neutralino $\chi$ obtained under various assumptions: universal scalar masses, also for Higgs multiplets, and the absence of CCB minima (UHM) or allowing them $\left(U H M_{\min }\right)$, and non-universal scalar masses ( $\mathrm{UUHM}$ ) [50].

olation. For example, as seen in Fig. 11, the h-HA mixing induced by $\mathrm{CP}$ violation may suppress the hZZ coupling, and the $h \bar{b} b$ coupling may also be suppressed, as seen in Fig. 12 [56]. We are currently re-evaluating the LEP lower limit on $m_{h}$, taking these effects into account. 


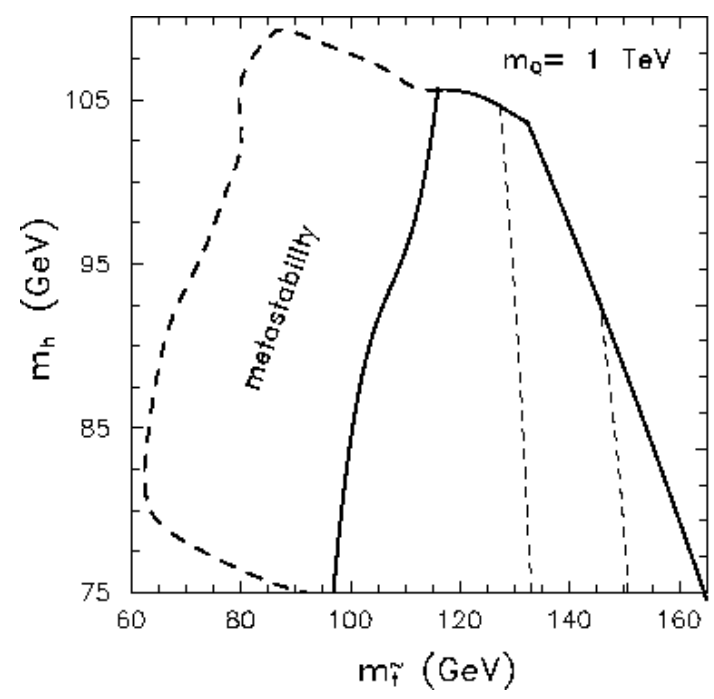

Figure 10: Region of the $m_{\tilde{t}}, m_{h}$ plane where the electroweak phase transition may be strongly first order [55].
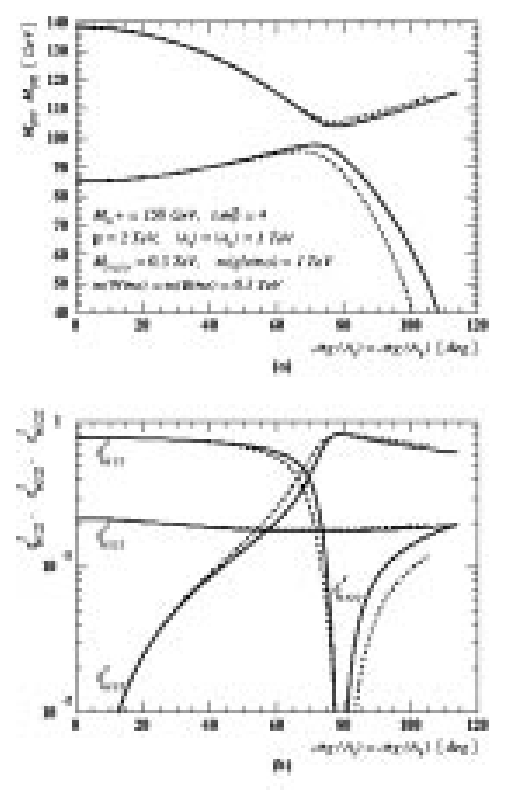

Figure 11: Illustration how CP violation in the MSSM may suppress the coupling of the lightest neutral Higgs boson to the $Z$ [56].

\section{Opportunities@ @uture Acceler- ators}

As discussed here by Fernandez [57], the LHC is under active construction, and is scheduled to start operating in 2005. In this talk, I concen-
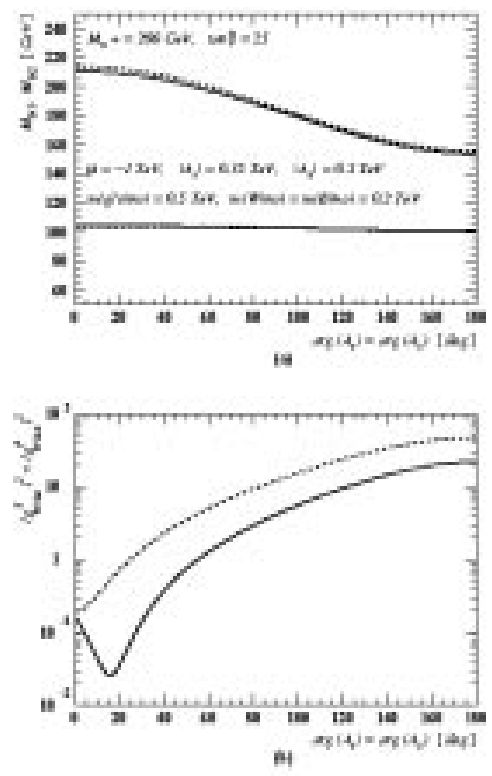

Figure 12: Illustration how CP violation in the MSSM may suppress the coupling of the lightest neutral Higgs boson to $\bar{b} b[56]$.

trate on two selected LHC physics topics, namely the quest for the Holy Higgs, and the search for supersymmetry. At low masses, the $H \rightarrow \bar{b} b$ and $\gamma \gamma$ decay signatures look the most promising, with $H \rightarrow 4 l^{ \pm}$over a large range of intermediate masses, and $H \rightarrow W^{+} W^{-} \rightarrow l^{+} \nu l^{-} \bar{\nu}, l^{ \pm} \nu j j$ and $H \rightarrow Z Z \rightarrow l^{+} l^{-} \bar{\nu} \nu$ interesting for high masses. As seen in Fig. 13 [58], there are no holes in the mass coverage, a couple of decay modes can normally be observed for any mass, and the Higgs mass can typically be measured with a precision $10^{-3} \lesssim \Delta m_{H} / m_{H} \lesssim 10^{-2}$. The LHC will also be able to discover supersymmetric Higgs bosons in two or more channels, over all the supersymmetric parameter space.

The LHC will produce principally stronglyinteracting sparticles, squarks $\tilde{q}$ and gluinos $\tilde{g}$, and they sould be detectable if they weigh $\lesssim 2$ $\mathrm{TeV}$, as seen in Fig. 14 [59]. This will enable the LHC to cover the parameter range allowed if the lightest supersymmetric particle provides the cold dark matter. The $\tilde{g}$ and $\tilde{q}$ often decay via complicated cascades, e.g., $\tilde{g} \rightarrow \tilde{b} \bar{b}, \tilde{b} \rightarrow \chi^{\prime} b, \chi^{\prime} \rightarrow$ $\chi l^{+} l^{-}$, which may be reconstructed to provide some detailed mass measurements, as indicated 


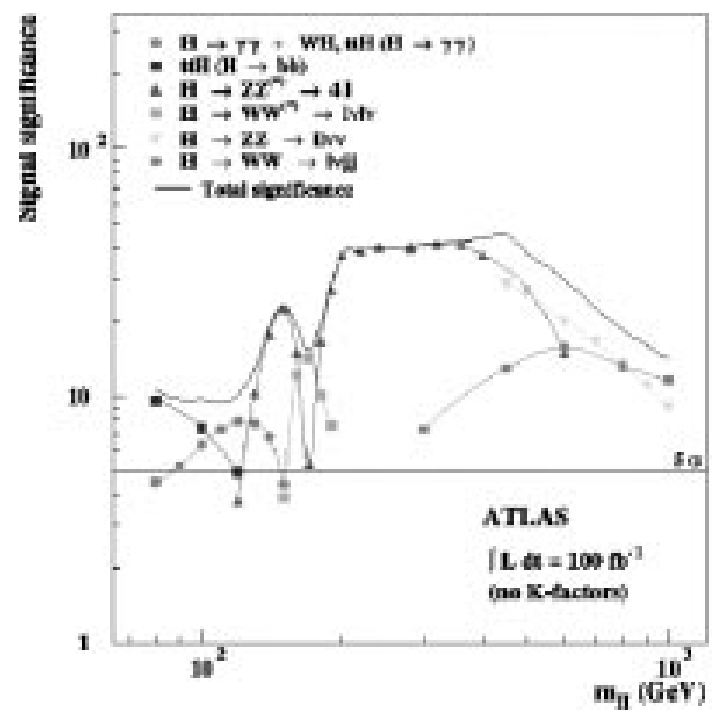

Figure 13: Significance of the Standard Model Higgs signal observable in the ATLAS experiment at the LHC [58], for the indicated value of the integrated luminosity.

in Fig. 15 [59].

A plausible scenario for physics after the LHC is that the Higgs will have been discovered, and one or two decay modes observed, and that several sparticles will have been discovered, but that heavier Higgses, charginos and sleptons may have escaped detection.

These lacunae provide some of the motivation for $e^{+} e^{-}$linear-collider (LC) physics. The very clean experimental environment, the egalitarian production of new weakly-interacting particles and the prospective availability of polarization make such a LC rather complementary to the LHC [60]. One of the big issues is what energy to choose for a first-generation LC: we know there is the $\bar{t} t$ threshold at $E_{c m} \simeq 350 \mathrm{GeV}$ and we believe there should be a $\mathrm{ZH}$ threshold at $E_{c m}=m_{Z}+m_{H} \lesssim 300 \mathrm{GeV}$. If one is above threshold, detailed studies of many Higgs decay modes (as seen in Fig. 16 [61]), or measurements of sparticle masses, become possible. However, we do not know what the supersymmetry threshold might be (assuming there is one!). For this reason, I think it is essential to retain as much flexibility as possible in the LC running energy.

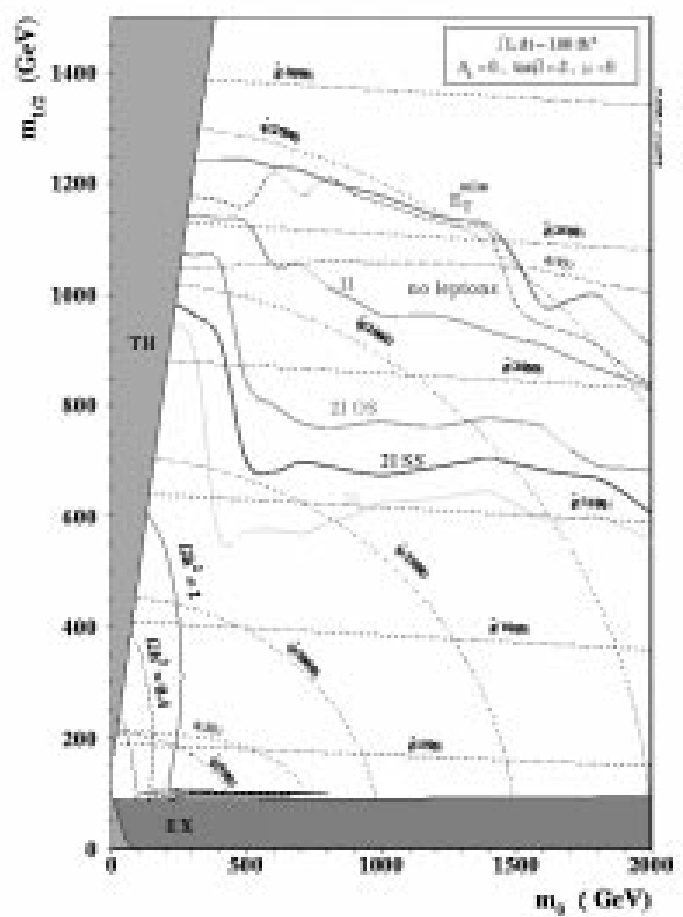

Figure 14: Regions of the $m_{0}, m_{1 / 2}$ plane accessible to different sparticle searches with the CMS experiment at the $\mathrm{LHC}$ [59].

In the meantime, the suggestion that the cold dark matter might consist of supersymmetric particles can be used [62] to guess the likelihood that a LC of given energy might find supersymmetry. As seen in Fig. 17, we find that all the dark matter parameter space can be explored if $E_{c m} \geq$ $1.25 \mathrm{TeV}$, and about $90 \%$ if $E_{c m}=1 \mathrm{TeV}$, but that an LC with $E_{c m}=0.5 \mathrm{TeV}$ would only cover about $60 \%$ of the dark matter parameter region.

My opinion is that physics will demand an $\mathrm{LC}$ in the $\mathrm{TeV}$ energy range, and I hope that the world can converge on a (single) project in this energy range. In the rest of this talk, I assume that an LC with $E_{c m} \sim 1 \mathrm{TeV}$ will be built somewhere in the world, and ask what other accelerator projects might be interesting [63].

One suggestion is a future larger hadron collider with $100 \mathrm{TeV} \lesssim E_{c m} \lesssim 200 \mathrm{TeV}$, that could explore the $10 \mathrm{TeV}$ mass region for the first time, if its luminosity rises to $10^{35} \mathrm{~cm}^{-2} \mathrm{~s}^{-1}$ or so. 


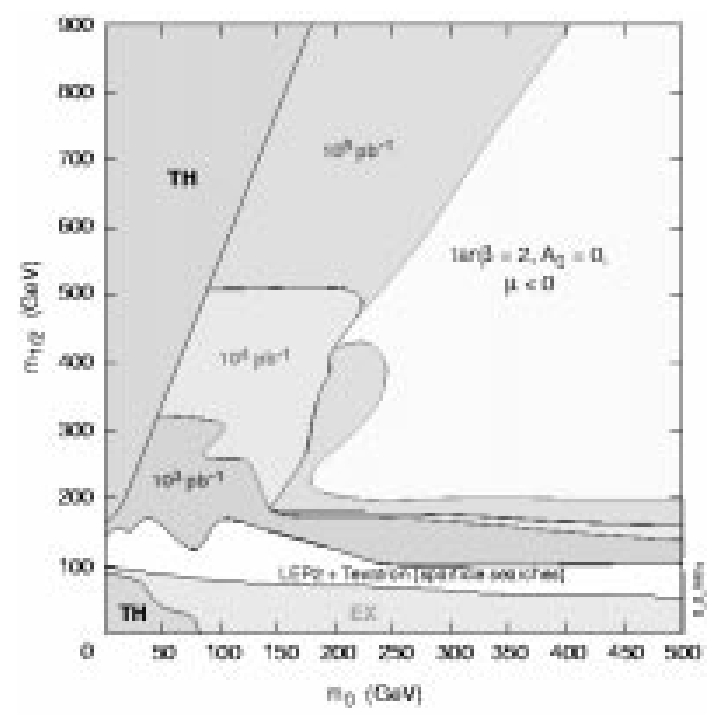

Figure 15: Regions of the $m_{0}, m_{1 / 2}$ plane in which cascade decays of sparticles are detectable with the CMS experiment at the $\mathrm{LHC}$ [59], for the indicated values of the integrated luminosity.

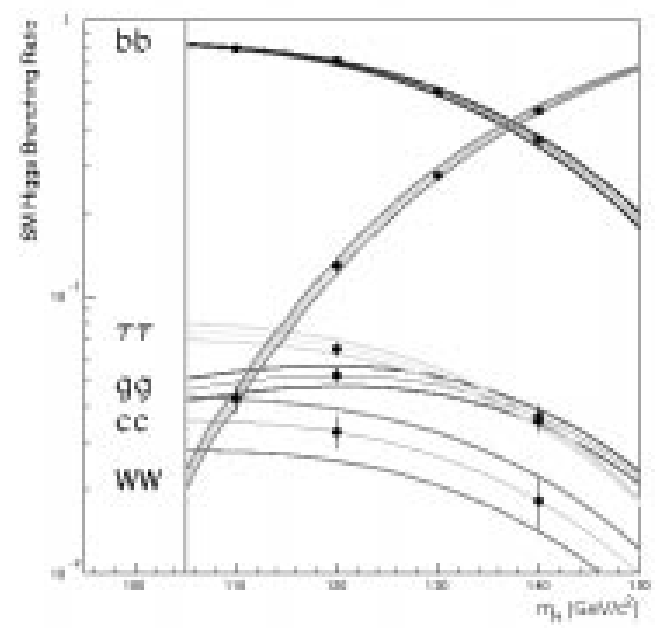

Figure 16: The Standard Model Higgs branching ratios may be determined very accurately at an $e^{+} e^{-}$ linear collider [61].

Such a machine is probably technically feasible, but it would be enormous, with a circumference of 100 to $500 \mathrm{~km}$. The principal challenge will be reducing the unit cost by an order of magnitude compared to the LHC. At present, we cannot formulate the physics questions for such a machine with great clarity.

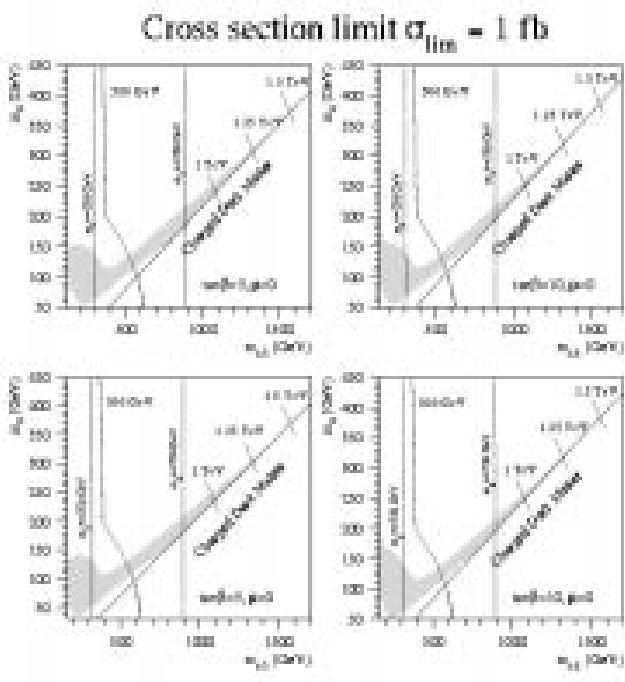

Figure 17: Regions of the $m_{1 / 2}, m_{0}$ plane consistent with cosmology (shaded) that may be explored with $e^{+} e^{-}$linear colliders of the specified energies [62], assuming universal soft supersymmetry-breaking scalar masses.

Another possibility is a higher-energy LC with $E_{c m} \gtrsim 2 \mathrm{TeV}$, capable, e.g., of making precise and complete studies of sparticle spectroscopy, or of any other electroweakly-interacting sparticles weighing $\lesssim 1 \mathrm{TeV}$. CERN is developing a potential technology for such an LC, called CLIC [64], in which an intense low-energy drive beam is used to provide $\mathrm{RF}$ to accelerate a more energetic but less intense colliding beam. Accelerating gradients in the range 100 to $200 \mathrm{MeV} \mathrm{m}^{-1}$ appear possible, enabling an LC with $E_{c m} \lesssim 5 \mathrm{TeV}$ to be accommodated in a tunnel $\sim 35 \mathrm{~km}$ long. The first physics study for such a machine was made at La Thuile in 1987 [65], and a new physics study has now been initiated [66]. Fig. 18 is a first result from this new study, showing what a $Z^{\prime}$ resonance might look like at CLIC [67].

A third type of accelerator currently attracting much interest is a complex of muon storage rings, as illustrated in Fig. 19. These could be developed in three steps [68]: first a neutrino factory in which muons are simply allowed to decay without colliding, secondly a Higgs factory colliding $\mu^{+} \mu^{-}$at $E_{c m} \sim m_{H}$, and thirdly a highenergy collider which might be compared with CLIC as a device to probe the high-energy fron- 


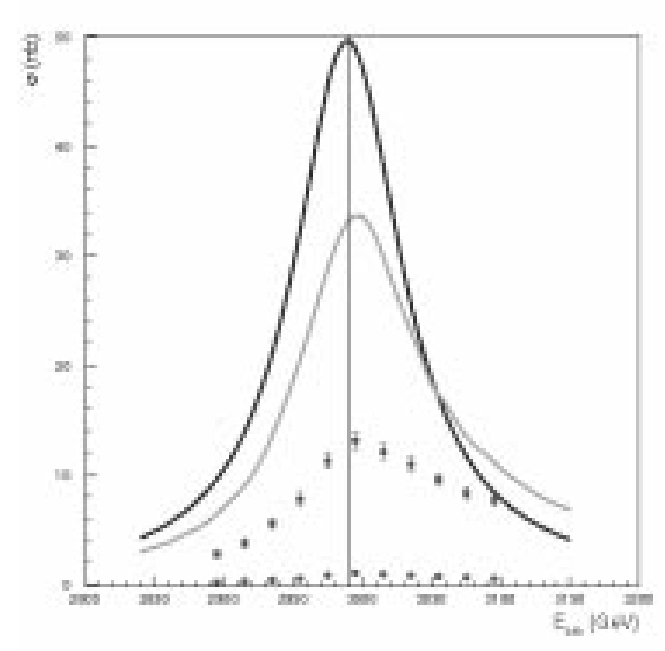

Figure 18: First impression of a possible $Z^{\prime}$ scan at $C L I C$, showing how beamstrahlung reduces the cross section below the value expected if only initial-state radiation is included [67].

tier. The chief advantages of neutrino beams from $\mu$ decays, as opposed to conventional beams from hadron decays, are their precisely calculable fluxes and spectra, and the facts that equal numbers of $\nu_{\mu}$ and $\bar{\nu}_{e}$ (or $\bar{\nu}_{\mu}$ and $\nu_{e}$ ) are produced. A $\mu^{+} \mu^{-}$collider used as a Higgs factory can measure very precisely the mass and width of any Higgs boson with mass around $100 \mathrm{GeV}$, distinguishing between the Standard Model and a superymmetric extension, and measurements of supersymmetric Higgs peaks could provide a unique window on $\mathrm{CP}$ violation. A $\mu^{+} \mu^{-}$collider at the high-energy frontier has advantages over an $e^{+} e^{-}$LC with similar energy, conferred by the more precise energy calibration and reduced energy spread, but the ultimate energy is limited by the neutrino radiation hazard. However, although it is very attractive, many technical problems need to be solved before the feasibility of such a muon storage rign complex can be established.

The basic concept for a neutrino factory involves a proton driver with beam power 1 to $20 \mathrm{MW}$, provided by either a linac or a rapidcycling synchrotron. This is used to produce pions, which decay into muons, of which about $0.1 /$ proton are cooled, accelerated to 10 to 50

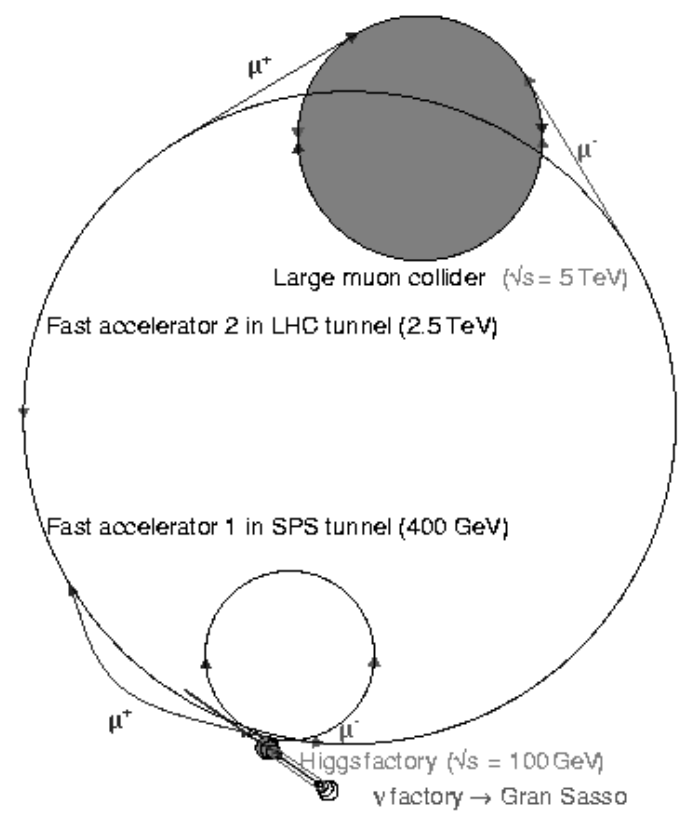

Figure 19: Artist's impression how a muon storage ring complex could be accommodated near CERN, including a $\nu$ factory, a Higgs factory and a high-energy muon collider [68].

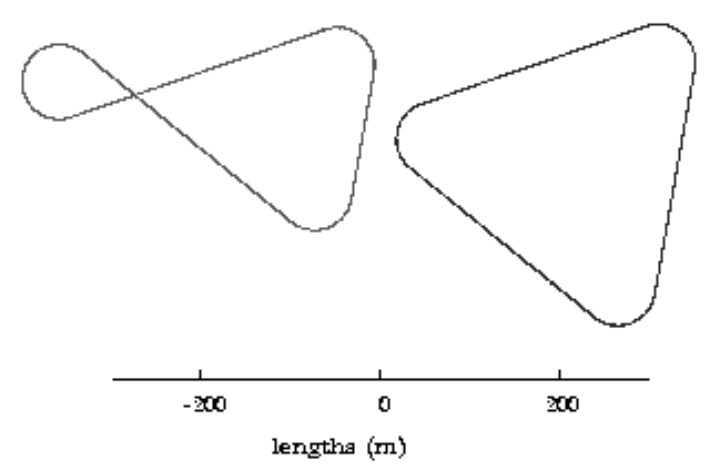

Figure 20: Vertical sections through possible designs of muon storage rings for $\nu$ factories: the re-entrant design is advantageous for retaining muon beam polarization [69].

$\mathrm{GeV}$, and stored in a ring. This need not be circular, and may look more like a bent paper-clip, as seen in Fig. 20 [69], with several straight sections sending $\sim\left(10^{20}\right.$ to $\left.10^{21}\right) \bar{\nu}_{\mu}, \nu_{e}$ per year each towards detectors at different distances.

In long-baseline neutrino experiments with a neutrino factory, the sensitivities to mixing angles and $\Delta m^{2}$ depend on $E_{\mu}$ and the distance $L$ [70]. As we see in Fig. 21, a $\nu_{\mu} \rightarrow \nu_{e}$ ap- 


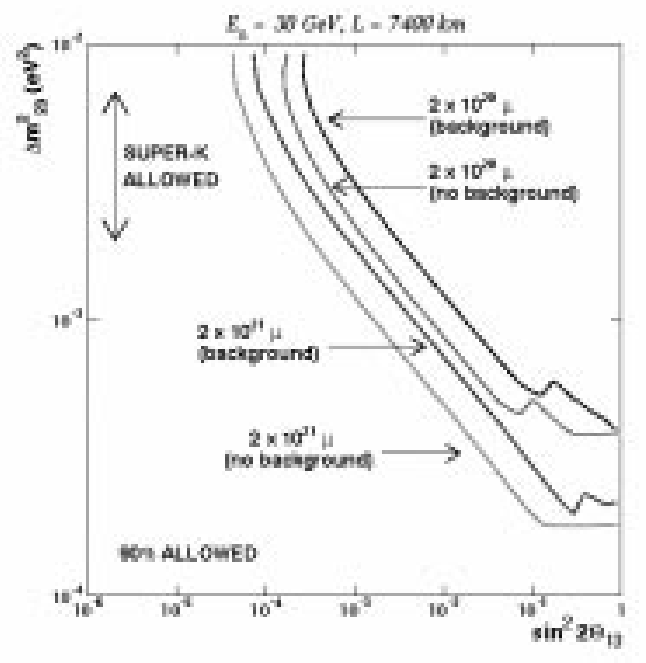

Figure 21: Sensitivities to $\nu_{\mu} \rightarrow \nu_{e}$ oscillations with a $\nu$ factory [71].

pearance experiment would be much more sensitive than the present Super-Kamiokande upper limit [71], or what may be achieved with MINOS. Moreover, as seen in Fig. 22, with sufficiently many $\mu$ decays one may be sensitive to $\mathrm{CP}$ violation and matter effects (which depend on the sign of $\Delta m^{2}$ ) in neutrino oscillations. For this, a detector at a distance of 2000 to $5000 \mathrm{~km}$ would be particularly advantageous. Ultimately, one could imagine a "World-Wide Neutrino Web" consisting of a $\nu$ factory in one region of the world feeding detectors in the same and other regions, as illustrated in Fig. 23.

Turning now to a Higgs factory, in the absence of a beam energy spread, the line shape (see Fig. 24) should be

$$
\sigma_{H}(s)=\frac{4 \pi \Gamma\left(H \rightarrow \mu^{+} \mu^{-}\right) \Gamma(H \rightarrow X)}{\left(s-m_{H}^{2}\right)^{2}+m_{H}^{2} \Gamma_{H}^{2}}
$$

It seems that it might be possible to reduce the beam energy spread to $\sim 0.01 \%$ or $10 \mathrm{MeV}$, comparable to the natural width of $3 \mathrm{MeV}$ for a Standard Model Higgs weighing about $100 \mathrm{GeV}$. Calibrating the beam energy via the $\mu^{ \pm}$polarization, it should then be possible to measure $m_{H}$ with a precision of $\pm 0.1 \mathrm{MeV}$, and the width to within $0.5 \mathrm{MeV}$, sufficient to distinguish a Standard Model Higgs boson from the lightest supersymmetric Higgs boson, over a large range
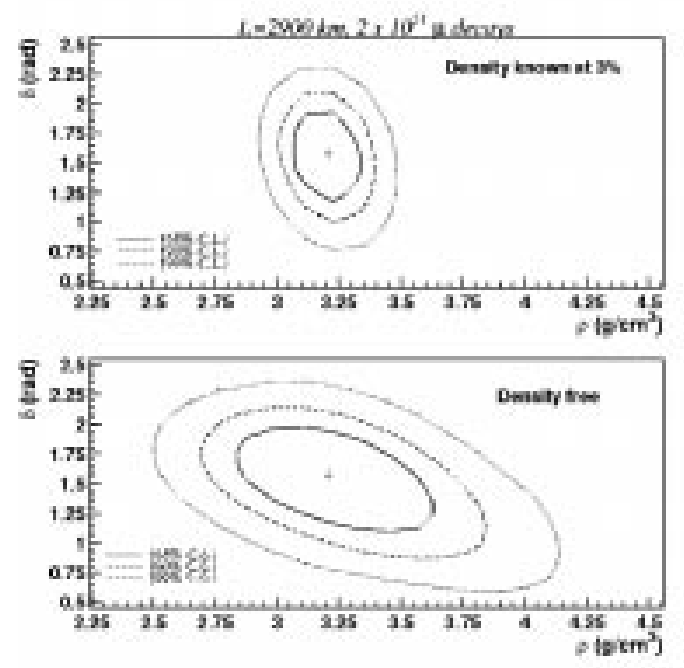

Figure 22: Sensitivity to CP violation in $\nu$ oscillations with a $\nu$ factory, either with or without knowledge of the Earth's matter density [71].

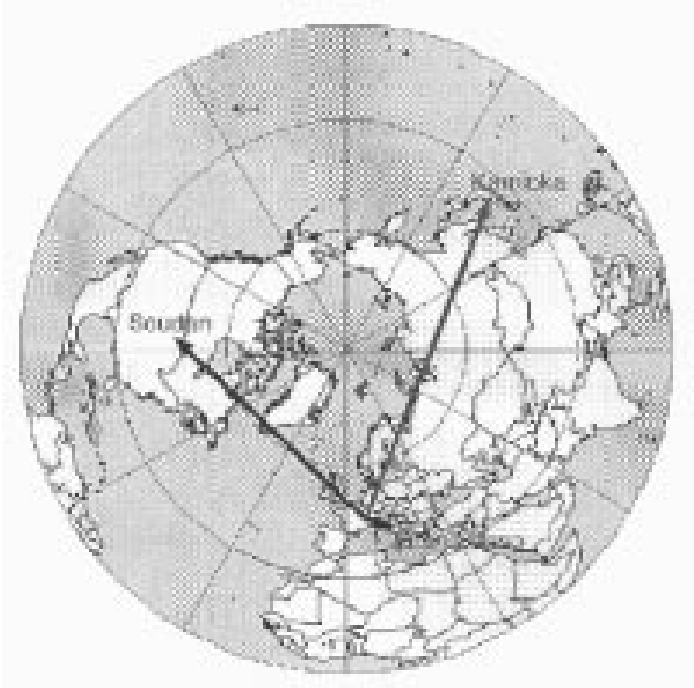

Figure 23: Artist's impression of a possible 'WorldWide Neutrino Web' of long-baseline $\nu$ beams aimed at experiments in different regions of the world.

of parameter space [68]. In the supersymmetric case, a second-generation Higgs factory able to explore the "Twin Peaks" of the $H$ and $A$ shown in Fig. 25 might be even more interesting, providing tests of $\mathrm{CP}$ symmetry analogous to those in the $K^{0}-\bar{K}^{0}$ system [72].

At the high-energy frontier, $\mu^{+} \mu^{-}$colliders would benefit from the absence of beamstrahlung 


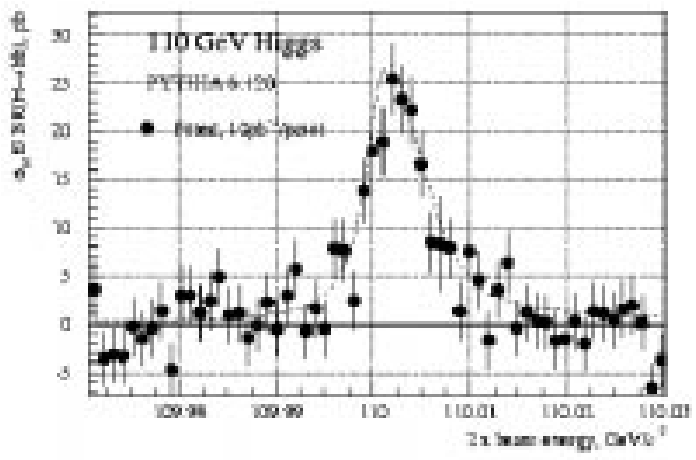

Figure 24: The possible line-shape of the Standard Model Higgs peak at a $\mu^{+} \mu^{-}$collider operated as a Higgs factory [68].

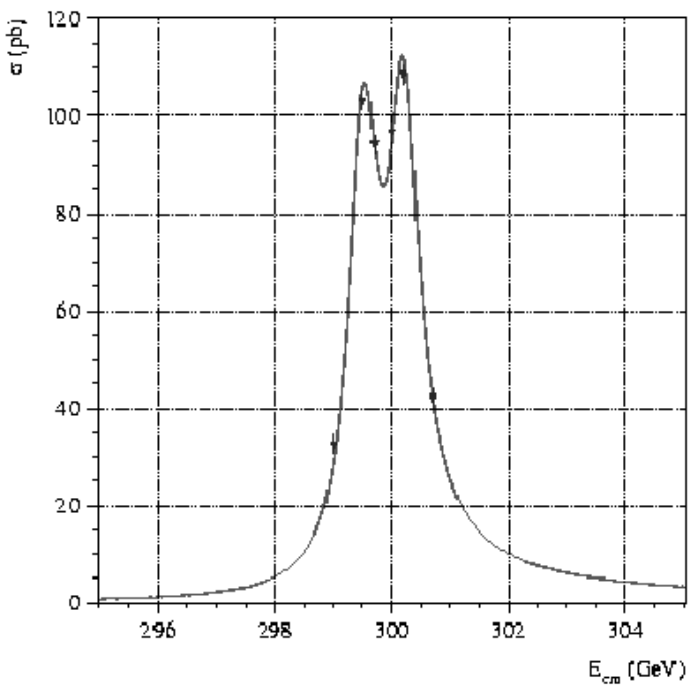

Figure 25: The possible line-shape of the twin MSSM Higgs peaks at a $\mu^{+} \mu^{-}$collider operated as a second-generation Higgs factory [68].

and reduced initial-state radiation, as compared to an $e^{+} e^{-}$LC such as CLIC. However, the latter offers controllable beam polarization, $e \gamma$ and $\gamma \gamma$ colliders "for free", and avoids the problems presented by $\mu$ decays. Moreover, an $e^{+} e^{-} \mathrm{LC}$ demonstrator, namely the SLC, has been built, whereas many of the technologies needed for a $\mu^{+} \mu^{-}$collider are at best glints in the eye, at present.

\section{Towards a Theory of Everything?}

The job description of a Theory of Everything
(TOE) is to unify all the fundamental interactions, including gravity, and to solve all the problems that arise in attempts to quantize gravitation. A possible solution is to replace point-like elementary particles by extended objects, and the first incarnation of this idea used one-dimensional closed loops of string. It was soon realized that this scenario requires extra space-time dimensions $10=4+6$ in the supersymmetric case, and/or extra interactions. The current reincarnation of this idea as $M$ theory includes other extended objects such as two-dimensional membranes, solids, etc. [73].

The key question is how to test these ideas. A popular suggestion is that the 6 surplus space dimensions are compactified on a Calabi-Yau manifold, but which one? 473800776 are known [74]! Recently we have embarked on a systematic study of Calabi-Yau (CY) spaces, constructed as zeroes of polynomials in weighted projective spaces, a technique which enables one to explore some of their internal properties and focus on these with desirable features [75]. Our harvest so far comprises $182737 \mathrm{CY}$ spaces, of which 211 have 3 generations and $\mathrm{K} 3$ fibrations as desired in some approaches to $M$ theory [75].

In the face of this ambiguity, how can one speak of phenomenological predictions from string theory? In fact, it has told us correctly that there cannot be more than 10 dimensions, that the gauge group cannot be very large, that matter representations cannot be very big, and that the top quark should not weigh more than about 190 $\mathrm{GeV}$. It has also provided a first-principles estimate of the unification scale, $M_{U} \sim$ few $\times 10^{17}$ $\mathrm{GeV}[76]$, which is not so far from the phenomenological bottom-up estimate of $(1$ or 2$) \times 10^{16} \mathrm{GeV}$.

Moreover, we now understand that all the different string theories are related by dualities in the general framework known as $M$ theory [77]. The question then becomes, in which part of its parameter space do we live? The GUT massscale calculation suggests that the string coupling may be strong [78], corresponding to one large dimension: $L \gg 1 / M_{G U T} \simeq 1 / 10^{16} \mathrm{GeV}$ $\gg 1 / m_{P} \equiv l_{P}$. 
Adventurous souls have then gone on to propose that one or more "small" dimensions might actually be rather large, perhaps $L \sim 1 \mathrm{TeV}^{-1}$ or even $\sim 1 \mathrm{~mm}$ [79]? In such models, there may be observable modifications of Newton's law: $G_{N} / r \rightarrow$ $G_{N}(1 / r)(L / r)^{\Delta D}$ at short distances, as well as possible new accelerator signatures. This suggestion offers plenty of phenomenological fun, but why should the scale of gravity sink so low? Are there any advantages for the hierarchy problem? So far, I have seen it reformulated, but not yet solved.

Before closing, I would like to mention a couple of radical possibilities for string phenomenology. As we heard here, surprisingly many ultrahigh-energy (UHE) cosmic rays have been observed above $E \sim 5 \times 10^{19} \mathrm{eV}$, more than expected above the GZK cutoff due to the reaction $p+\gamma_{C M B} \rightarrow \Delta^{+}[80]$. Unless one modifies relativistic kinematics (see later) these UHE cosmic rays should have originated nearby, at distances $d \lesssim 100 \mathrm{Mpc}$ for $E \sim 10^{20} \mathrm{eV}$, but no discrete sources have yet been confirmed.

Might they originate from the decays of supermassive dark matter particles? It has recently been realized that such particles weighing $10^{10}$ $\mathrm{GeV}$ or more might have been produced by nonthermal mechanisms early in the history of the Universe [81]. Possible candidates for these unstable heavy relics can be found in string theory, particularly as bound states in the hidden sector, which we have termed cryptons [82]. They could well have masses $\sim 10^{13} \mathrm{GeV}$ and be metastable, decaying via higher-dimensional operators into multiple leptons and quarks. Simulations indicate that they could well produce the observed UHE tail of the cosmic-ray spectrum, as seen in Fig. 26 [83]. The Auger project should be able to tell us whether this mechanism is tenable [80].

Even more speculative is the suggestion of quantum-gravitational phenomenology. Might the space-time foam of quantum-gravitational fluctuations in the fabric of space induce quantum decoherence and/or CPT violation at the microscopic scale [84]? Here the most sensitive probe may be the $K^{0}-\bar{K}^{0}$ system [85]. Might the velocity of light [86] (or a neutrino [87]) depend

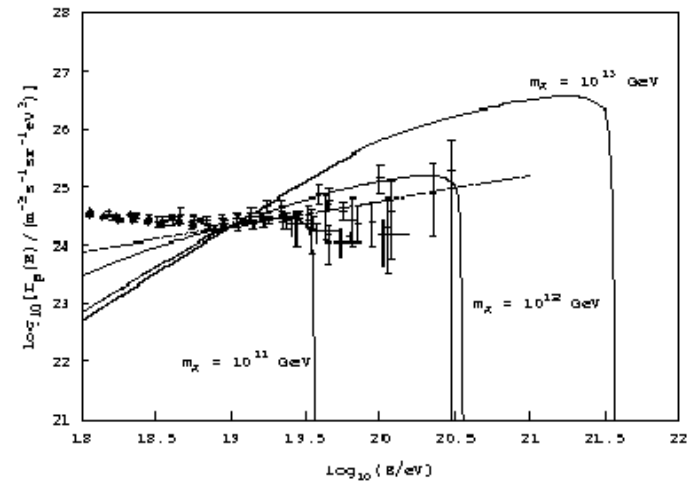

Figure 26: Comparison between data on ultra-highenergy cosmic rays around the GZK cutoff with a simulation of crypton decays [83].

on its energy, because of recoil effects on the space-time vacuum? Here the most sensitive direct tests may be provided by distant, energetic sources with short time-scales, such as gammaray bursters (GRBs) [88], active galactic nuclei [89] and pulsars [90], and some such models are also constrained by the kinematics of UHE cosmic rays [91]. Fig. 27 shows fits to BATSE data on GRB 970508 in different energy channels. A regression analysis of fits to GRBs with measured redshifts has been used to constrain any possible energy dependence of the velocity of light: $\delta c / c \leq E / M: M>10^{15} \mathrm{GeV}[92]$.

\section{Final Comments}

The history of physics reveals many ways in which it may advance, being driven either by pure theoretical thought or by experimental breakthroughs. Pure theoretical speculation must in any case be tempered by experimental reality: we can never forget that in physics, as any other science, experiment is the ultimate arbiter. Particle physics is currently fortunate. On the one hand, experiments at LEP and elsewhere have shown that the Standard Model is a solid rock on which to build. On the other hand, experiments on neutrinos strongly indicate oscillations, and hence physics beyond the Standard Model. There are exciting new experimental programmes underway to explore the flavour problem, to pin down models 


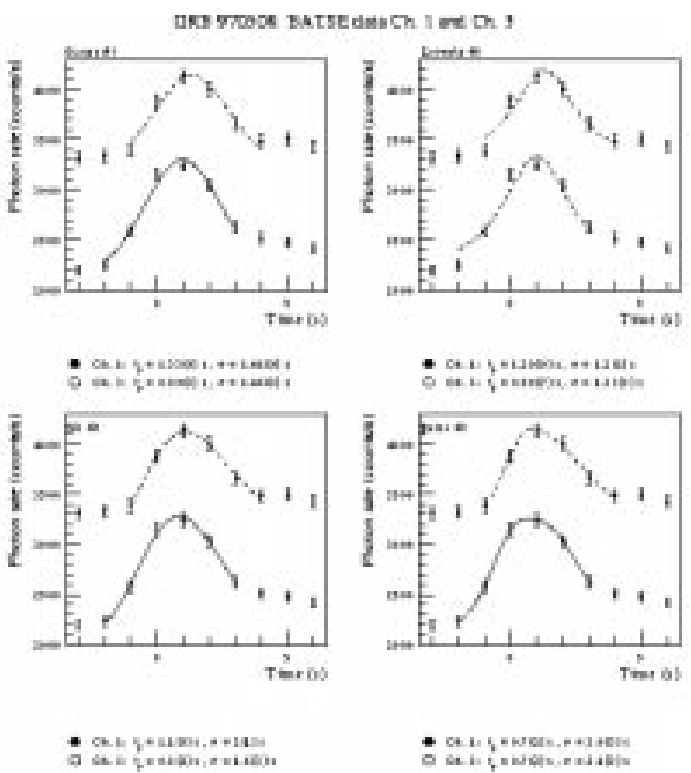

Figure 27: Fits to BATSE data on GRB 970508 in different energy channels [92]. Comparisons between the arrival times of the peaks at different energies are used to constrain any possible energy dependence of the velocity of light.

of neutrino oscillations, and to explore the $\mathrm{TeV}$ energy range.

Beyond our daily concerns, we have the great unsolved problem of twentieth-century physics left to stimulate us: reconcile gravity and quantum mechanics. Great theoretical advances towards this goal have been made during recent years, but we do not know how far we are from this goal. In particular, we have not yet defined a clear experimental test that will confirm or refute string theory. Finding it is our key phenomenological challenge.

\section{References}

[1] D. Abbaneo et al., LEP Electroweak Working Group, http://lepewwg.web.cern.ch/LEPEWWG/ Welcome.html; CERN-EP/2000-016 (2000).

[2] F. Behner, talk at this meeting.

[3] J. Nowell, talk at this meeting.

[4] P. Bock et al., LEP Working Group for Higgs boson searches, CERN-EP-2000-055 (2000).

[5] D. Smith, talk at this meeting.
[6] E. Gross and A. L. Read, Talk at the 14th Rencontres de Physique de la Valle d'Aoste: Results and Perspectives in Particle Physics, La Thuile, Valle d'Aoste, Italy, $27 \mathrm{Feb}$ - $4 \mathrm{Mar}$ 2000, CERN-EP/2000-034.

[7] J. Ellis, G. Ridolfi and F. Zwirner, Phys. Lett. B257 (1991) 83; M.S. Berger, Phys. Rev. D41 (1990) 225; Y. Okada, M. Yamaguchi and T. Yanagida, Prog. Theor. Phys. 85 (1991) 1; Phys. Lett. B262 (1991) 54; H.E. Haber and R. Hempfling, Phys. Rev. Lett. 66 (1991) 1815; R. Barbieri, M. Frigeni and F. Caravaglios, Phys. Lett. B258 (1991) 167.

[8] LEP Physics Coordination, P. Janot, http://alephwww.cern.ch/ janot/LEPCO/.

[9] For the latest update, see: http://sl.web.cern.ch/SL/opnews/stat/ luday.gif.

[10] M. Carena, S. Mrenna and C. E. Wagner, hep$\mathrm{ph} / 9907422$.

[11] Y. Nir, hep-ph/9911321, and lectures at this meeting.

[12] A. Alavi-Harati et al., KTeV Collaboration, Phys. Rev. Lett. 83 (1999) 22; S. Taegar, talk at this meeting.

[13] V. Fanti et al., NA48 Collaboration, Phys. Lett. B465 (1999) 335.

[14] M. Kobayashi and T. Maskawa, Prog. Theor. Phys. 49 (1973) 652.

[15] A. J. Buras, hep-ph/9908395; S. Bertolini, J. O. Eeg and M. Fabbrichesi, hep-ph/0002234; M. Ciuchini and G. Martinelli, hep-ph/0006056; and references therein.

[16] A. Ceccucci, for the NA 48 Collaboration, CERN seminar,

http://na48. web.cern.ch/NA48/Welcome.html; $\mathrm{J}$ Ocariz, talk at this meeting.

[17] A. Masiero and O. Vives, hep-ph/0001298; A. Masiero, talk at this meeting.

[18] A. Ali and D. London, hep-ph/0002167.

[19] K. Ackerstaff et al., OPAL Collaboration, Eur. Phys. J. C5 (1998) 379.

[20] T. Affolder et al., CDF Collaboration, Phys. Rev. D61 (2000) 072005.

[21] ALEPH Collaboration, contactperson R. Forty, ALEPH 99-099, CONF 99-054 (1999).

[22] D. Cronin-Hennessy et al., CLEO Collaboration, hep-ex/0001010. 
[23] R. Barbieri, J. Ellis and M. K. Gaillard, Phys. Lett. B90 (1980) 249.

[24] M. Gell-Mann, P. Ramond and R. Slansky, Proceedings of the Stony Brook Supergravity Workshop, New York, 1979, eds. P. Van Nieuwenhuizen and D. Freedman (North-Holland, Amsterdam).

[25] Z. Maki, M. Nakagawa and S. Sakata, Prog. Theor. Phys. 28 (1962) 247.

[26] W. Hu, D. J. Eisenstein and M. Tegmark, Phys. Rev. Lett. 80 (1998) 5255 and http://www.sns.ias.edu/ whu/.

[27] L. Baudis et al., Phys. Rev. Lett. 83 (1999) 41.

[28] J. Ellis and S. Lola, Phys. Lett. B458 (1999) 310; N. Haba, Y. Matsui, N. Okamura and T. Suzuki, hep-ph/0005064 and references therein; J. A. Casas, J. R. Espinosa, A. Ibarra and I. Navarro, Nucl. Phys. B573 (2000) 652 and references therein; P. H. Chankowski, W. Krolikowski and S. Pokorski, Phys. Lett. B473 (2000) 109.

[29] R. Barbieri, G. G. Ross and A. Strumia, JHEP 9910 (1999) 020; E. Ma, J. Phys. G G25 (1999) L97.

[30] A. Smirnov, lectures at this meeting.

[31] Y. Fukuda et al., Super-Kamiokande Collaboration, Phys. Lett. B467 (1999) 185, and references therein; H. Sobel, for the SuperKamiokande collaboration, talk at $\nu$ 2000, Sudbury, Canada, http://nu2000.sno.laurentian.ca/;

Y. Totsuka, for the Super-Kamiokande collaboration, talk at SUSY2K, CERN, Geneva, Switzerland,

http://wwwth.cern.ch/susy2k/

susy $2 \mathrm{kf}$ inalprog.html.

[32] J. N. Bahcall, P. I. Krastev and A. Y. Smirnov, Phys. Lett. B477 (2000) 401, and hep$\mathrm{ph} / 0002293$.

[33] M. Apollonio et al., Chooz Collaboration, Phys. Lett. B466 (1999) 415; F. Boehm et al., Palo Verde Collaboration, hep-ex/0003022.

[34] K. Ishihara, Ph. D. thesis Study of $\nu_{\mu} \rightarrow \nu_{\tau}$ and

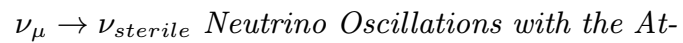
mospheric Data in Super-Kamiokande, ICRRReport-457-2000-1;

H. Sobel and Y. Totsuka, in [31].

[35] Y. Suzuki, for the Super-Kamiokande collaboration, talk at $\nu$ 2000, Sudbury, Canada, http://nu2000.sno.laurentian.ca/.
[36] J. Boger et al., SNO Collaboration, nuclex/9910016; A. McDonald, for the SNO collaboration, talk at $\nu 2000$, Sudbury, Canada, http://nu2000.sno.laurentian.ca/.

[37] M. C. Chen, for the BOREXINO Collaboration, Prepared for 29th International Conference on High-Energy Physics (ICHEP 98), Vancouver, British Columbia, Canada, 23-29 Jul 1998.

[38] K. Nakamura, for the K2K collaboration, talk at $\nu$ 2000, Sudbury, Canada, http://nu2000.sno.laurentian.ca/. These results update those found in: Y. Oyama, for the K2K Collaboration, Talk at the XXXV Rencontres de Moriond, Electroweak Interactions and Unified Theories, Les Arcs, March 1118 2000, hep-ex/0004015.

[39] A. Suzuki, for the KamLAND Collaboration, Nucl. Phys. Proc. Suppl. 77 (1999) 171.

[40] E. Ables et al., MINOS Collaboration, $P$ 875: A long baseline neutrino oscillation experiment at Fermilab, FERMILAB-PROPOSAL-P875 (1995).

[41] G. Acquistapace et al., The CERN neutrino beam to Gran Sasso (NGS): Conceptual technical design, CERN-98-02 (1998).

[42] K. Kodama et al., OPERA Collaboration, Progress Report CERN/SPSC 99-20, SPSC/M635, LNGS-LOI 19/99 (1999).

[43] F. Arneodo et al., ICANOE Collaboration, Proposal LNGS-P21/99 INFN/AE-99-17, CERN/SPSC 99-25, SPSC/P314.

[44] L. Maiani, Proceedings of the Summer School on Particle Physics, Gif-sur-Yvette, 1979, (IN2P3, Paris, 1980), p.3; G. 't Hooft, in Recent developments in Field Theories, eds. G. 't Hooft et al., (Plenum Press, New York, 1980); E. Witten, Nucl. Phys. B188 (1981) 513; R. K. Kaul, Phys. Lett. 109B (1982) 19.

[45] J. Ellis, S. Kelley and D. V. Nanopoulos, Phys. Lett. B249 (1990) 441 and Phys. Lett. B260 (1991) 131; U. Amaldi, W. de Boer and H. Furstenau, Phys. Lett. B260 (1991) 447; C. Giunti, C. W. Kim and U. W. Lee, Mod. Phys. Lett. A6 (1991) 1745; P. Langacker and M. Luo, Phys. Rev. D44 (1991) 817.

[46] P. H. Chankowski, J. Ellis, M. Olechowski and S. Pokorski, Nucl. Phys. B544 (1999) 39; L. Giusti, A. Romanino and A. Strumia, Nucl. Phys. B550 (1999) 3; J. L. Feng, K. T. Matchev and T. Moroi, Phys. Rev. D61 (2000) 075005. 
[47] J. Ellis, J. S. Hagelin, D. V. Nanopoulos, K. Olive and M. Srednicki, Nucl. Phys. B238 (1984) 453.

[48] D. Fouchez, talk at this meeting.

[49] E. Roulet, astro-ph/0004342, talk at this meeting.

[50] J. Ellis, T. Falk, G. Ganis and K. A. Olive, hep$\mathrm{ph} / 0004169$.

[51] J. Ellis, T. Falk and K. A. Olive, Phys. Lett. B444 (1998) 367; J. Ellis, T. Falk, K. A. Olive and M. Srednicki, Astropart. Phys. 13 (2000) 181.

[52] G. Kane, lectures at this meeting; see also G. L. Kane and L. Wang, hep-ph/0003198.

[53] M. Losada, talk at this meeting

[54] E. Ma, talk at this meeting, and hep$\mathrm{ph} / 0001246$.

[55] M. Carena, M. Quiros and C. E. Wagner, Nucl. Phys. B524 (1998) 3.

[56] M. Carena, J. Ellis, A. Pilaftsis and C. E. Wagner, hep-ph/0003180.

[57] E. Fernandez, lectures at this meeting.

[58] ATLAS Collaboration, ATLAS Detector and Physics Performance Technical Design Report, http://atlasinfo.cern.ch/Atlas/GROUPS/PHYSICS/TDR/access.html.

[59] S. Abdullin and F. Charles, Nucl. Phys. B547 (1999) 60.

[60] E. Accomando et al., ECFA/DESY LC Physics Working Group, Phys. Rept. 299 (1998) 1.

[61] M. Battaglia, hep-ph/9910271.

[62] J. Ellis, G. Ganis and K. A. Olive, Phys. Lett. B474 (2000) 314.

[63] J. Ellis, E. Keil and G. Rolandi, Options for future colliders at CERN, CERN-EP/98-03 (1998).

[64] J.-P. Delahaye, for the CLIC study team, The CLIC Study of a Multi-TeV $e^{ \pm}$Linear Collider, CERN-PS/99-005 (1999),

http://cern.web.cern.ch/CERN/Divisions/$\mathrm{PS} / \mathrm{CLIC} /$ Welcome.html.

[65] Proceedings of the Workshop on Physics at Future Accelerators, La Thuile, Italy and Geneva, Switzerland, Jan 7-13, 1987, ed. J.H. Mulvey, CERN-87-07 (1987).

[66] Organizing/steering group, M. Battaglia, A. De Roeck, C. Detraz, J. Ellis, D. Schulte, R. Settles, G. Wilson, http://clicphysics . web.cern.ch/CLICphysics/.
[67] M. Battaglia, Talk at first general open meeting for CLIC Physics Studies, May 25 - 26, 2000, http://clicphysics.web.cern. ch/CLICphysics/.

[68] Prospective study of muon storage rings at $C E R N$, eds. B. Autin, A. Blondel and J. Ellis, CERN-99-02 (1999).

[69] K.T. McDonald et al., the Neutrino Factory and Muon Collider Collaboration, Expression of Interest in RED towards a Neutrino Factory Based on a Storage Ring and a Muon Collider, physics/9911009.

[70] A. De Rujula, M. B. Gavela and P. Hernandez, Nucl. Phys. B547 (1999) 21; S. Dutta, R. Gandhi and B. Mukhopadhyaya, hepph/9905475; M. Tanimoto, Phys. Lett. B462 (1999) 115; A. Romanino, Nucl. Phys. B574 (2000) 675; V. Barger, S. Geer, R. Raja and K. Whisnant, Phys. Rev. D62 (2000) 013004; M. Freund, M. Lindner, S. T. Petcov and A. Romanino, Nucl. Phys. B578 (2000) 27; A. Cervera, A. Donini, M. B. Gavela, J. J. Gomez Cadenas, P. Hernandez, O. Mena and S. Rigolin, hep-ph/0002108; I. Mocioiu and R. Shrock, hep-ph/0002149; V. Barger, S. Geer, R. Raja and K. Whisnant, hepph/0003184; A. Blondel et al., CERN-EP-2000053; M. Freund, P. Huber and M. Lindner, hepph/0004085; V. Barger, S. Geer, R. Raja and K. Whisnant, hep-ph/0004208; O. Yasuda, hepph/0005134; C. Albright et al., FERMILABFN-692; K. Dick, M. Freund, P. Huber and M. Lindner, hep-ph/0006090.

[71] A. Bueno, M. Campanelli and A. Rubbia, hep$\mathrm{ph} / 0005007$.

[72] B. Grzadkowski, hep-ph/0005170, and references therein.

[73] J. Russo, lectures at this meeting.

[74] M. Kreuzer and H. Skarke, hep-th/0002240.

[75] F. Anselmo, J. Ellis, D. V. Nanopoulos and G. Volkov, hep-th/0002102, and hepth/0007115.

[76] See, for example, I. Antoniadis, J. Ellis, R. Lacaze and D. V. Nanopoulos, Phys. Lett. B268 (1991) 188.

[77] C. P. Bachas, hep-ph/0003259, and references therein.

[78] E. Witten, Nucl. Phys. B471 (1996) 135; P. Horava and E. Witten, Nucl. Phys. B460 (1996) 506 and Nucl. Phys. B475 (1996) 94. 
[79] I. Antoniadis and K. Benakli, hep-ph/0004240, and references therein.

[80] A. Zepeda, talk at this meeting; C. K. Guerard, for the Pierre Auger Collaboration, Nucl. Phys. Proc. Suppl. 75A (1999) 380.

[81] E. W. Kolb, D. J. Chung and A. Riotto, hep$\mathrm{ph} / 9810361$, and references therein.

[82] J. Ellis, J. L. Lopez and D. V. Nanopoulos, Phys. Lett. B247 (1990) 257; J. Ellis, G. B. Gelmini, J. L. Lopez, D. V. Nanopoulos and S. Sarkar, Nucl. Phys. B373 (1992) 399; K. Benakli, J. Ellis and D. V. Nanopoulos, Phys. Rev. D59 (1999) 047301.

[83] M. Birkel and S. Sarkar, Astropart. Phys. 9 (1998) 297; S. Sarkar, hep-ph/0005256, and references therein.

[84] J. Ellis, N. E. Mavromatos and D. V. Nanopoulos, Gen. Rel. Grav. 31 (1999) 1257, and grqc/0005100.

[85] J. Ellis, J. S. Hagelin, D. V. Nanopoulos and M. Srednicki, Nucl. Phys. B241 (1984) 381.

[86] G. Amelino-Camelia, J. Ellis, N. E. Mavromatos and D. V. Nanopoulos, Int. J. Mod. Phys. A12 (1997) 607.

[87] J. Alfaro, H. A. Morales-Tecotl and L. F. Urrutia, Phys. Rev. Lett. 84 (2000) 2318; J. Ellis, N. E. Mavromatos, D. V. Nanopoulos and G. Volkov, gr-qc/9911055; L. Urrutia, talk at this meeting.

[88] G. Amelino-Camelia, J. Ellis, N. E. Mavromatos, D. V. Nanopoulos and S. Sarkar, Nature 393 (1998) 763.

[89] S. D. Biller et al., Phys. Rev. Lett. 83 (1999) 2108.

[90] P. Kaaret, astro-ph/9903464.

[91] L. Gonzalez-Mestres, physics/0003080, and references therein.

[92] J. Ellis, K. Farakos, N. E. Mavromatos, V. A. Mitsou and D. V. Nanopoulos, astroph/9907340. 